\title{
INFLUÊNCIA DOS ATRIBUTOS DO SOLO SOBREAQUALIDADE DA MADEIRA DE Pinus taeda PARA PRODUÇÃO DE CELULOSE KRAFT ${ }^{1}$
}

\author{
Patrícia Aparecida Rigatto ${ }^{2}$, Renato Antônio Dedecek ${ }^{3}$ e Jorge Luis Monteiro de Matos ${ }^{4}$
}

\begin{abstract}
RESUMO - Neste trabalho foram analisados os efeitos dos atributos do solo sobre a qualidade da madeira de Pinus taeda para produção de celulose Kraft, em áreas da Klabin, em Telêmaco Borba-PR. Foram estudados oito sítios com árvores de 12 anos de idade, selecionados pelo tipo de solo, textura e vegetação primária. Para caracterização dos sítios foram realizadas coletas de amostras em três horizontes, tendo sido coletadas amostras indeformadas e compostas, analisando-se as seguintes variáveis no solo: densidade global, porosidade total, macroporosidade, disponibilidade de água, fertilidade e granulometria. Selecionaram-se cinco árvores médias por sítio, nas quais foram medidos as alturas total e comercial e o DAP e retirados discos, sendo este material ensaiado quanto a densidade básica, composição química, características morfológicas dos traqueídeos e produção de celulose Kraft. Com relação às propriedades da madeira, os atributos físicos do solo demonstraram ter maior influência. De modo geral, as madeiras provenientes de sítios com texturas mais argilosas apresentaram menores valores de densidade básica; maiores teores de extrativos e lignina; menores teores de holocelulose e celulose; traqueídeos mais curtos, mais largos, com paredes mais finas e com diâmetros do lúmen maiores; e menor rendimento em celulose. A partir destes resultados, concluiu-se ser possível a previsão de propriedades da polpa através da análise das características da madeira associada às condições edáficas reinantes.
\end{abstract}

Palavras-chave: Fertilidade do solo, atributos do solo, anatomia da madeira e química da madeira.

\section{INFLUENCE OF SOIL ATTRIBUTES ON QUALITY OF Pinus taeda WOOD FOR CELLULOSE KRAFT PRODUCTION}

\begin{abstract}
To meet the increasing demand for forest products, much of the future timber supply will come from trees grown in managed plantations. This work was carried out to analyze the effects of soil attributes on Pinus taeda wood quality to produce Kraft cellulose at Klabin Parana Cellulose Co., in Telêmaco Borba, PR, Brazil. The study focused on the influence of soil attributes on the anatomical, physical and chemical wood characteristics, as well as their relation to cellulose production. Eight sites with twelve-year-old trees were selected based on soil type, soil texture and primary vegetation. The soil variables studied were bulk density, total porosity, macroporosity, water availability, fertility and grain size distribution. For tree selection, dendrometric measurements of 50 trees were taken per site, and 5 average size trees selected per site. Total and commercial height and DBH were measured and discs removed. Tree samples were analyzed for basic density, chemical composition, tracheid morphological characteristics, nutritional status and Kraft cellulose production. The soil chemical attributes exerted significant influence on wood quality, but the soil physical attributes showed greater influence on cellulose productivity. Sites with higher growth rate and those with clay texture showed lower values of basic density, higher content of extract and lignin, thus showing lower content of holocellulose and cellulose, shorter and wider tracheids with thinner walls and higher lumen diameters, and lower cellulose yield. Based on these results, it was concluded that cellulose pulp characteristics can be estimated by analyzing wood characteristics combined with the existing site edaphic conditions.
\end{abstract}

Key words: $\quad$ Soil fertility, soil attributes, wood anatomy and wood chemistry.

Recebido para publicação em 5.12.2002 e aceito para publicação em 30.4.2004.

Parte da Dissertação de Mestrado desenvolvida na Universidade Federal do Paraná.

2 Engenheira Florestal/Pós-graduanda em Agronomia, Área de concentração em Ciência do Solo, Departamento de Solos e Engenharia Agrícola da Universidade Federal do Paraná - UFPR, Rua dos Funcionários 1540, 80035-050 Curitiba-PR, <patyrigatto@yahoo.com.br>. ${ }^{3}$ Engenheiro-Agrônomo, Ph.D., Pesquisador do Centro Nacional de Pesquisa de Florestas Embrapa, Estrada da Ribeira, Km 111, Caixa Postal 319, 83411-000 Colombo-PR, <dedecek@ cnpf.embrapa.br>. ${ }^{4}$ Engenheiro Florestal, Prof. Dr., Departamento de Engenharia e Tecnologia Florestal da UFPR, Rua Lothario Meissner 3400, 80210-170 Curitiba-PR,<jmatos@floresta.ufpr.br>. 


\section{INTRODUÇÃO}

A crescente demanda de celulose e papel e a globalização de seus mercados vêm atuando como importantes fatores de estímulo à busca de matérias-primas mais adequadas às suas fabricações. No Brasil, a madeira de Pinus é um exemplo típico dessa tendência, o que pode ser constatado através da intensidade de estudos com espécies desse gênero (Birkland, 1990).

$\mathrm{O}$ aumento da capacidade produtiva das indústrias de polpa e a crescente exigência do mercado consumidor, principalmente o externo, quanto à qualidade da polpa produzida, conduziram a um novo conceito de seleção dos sítios para formação das florestas, buscando-se maior homogeneidade da matéria-prima a ser utilizada pela indústria. A seleção por qualidade da madeira, atualmente, leva em consideração os efeitos das propriedades físicas e químicas da madeira sobre o processo de deslignificação e na qualidade da polpa produzida (Sansígolo, 2000).

Para o setor celulósico-papeleiro, o processo de avaliação de qualidade não se limita exclusivamente à análise de qualidade do produto. Visando aumentar a eficiência desse processo, são analisados todos os aspectos que possam influenciar a sua qualidade final, pois na melhor qualidade da matéria-prima reside parte significativa da qualidade do produto (Clarke \& Wessels, 1995).

Os atributos do solo são um dos fatores que podem influenciar a qualidade final da polpa produzida, através de sua interferência na taxa de crescimento dos sítios florestais e, conseqüentemente, na qualidade da madeira produzida.

Uma questão a ser elucidada é se estas interferências na taxa de crescimento geradas pelos atributos do solo podem resultar em madeira de baixa qualidade e, conseqüentemente, menor qualidade final da polpa produzida.

Ainda são poucos os estudos que relacionam diretamente a interferência dos atributos do solo sobre a qualidade da madeira de espécies florestais; o que existe são trabalhos relacionando a taxa de crescimento, que é o resultado destes atributos, com a qualidade da madeira e, conseqüentemente, suas interações com o rendimento e a qualidade da celulose produzida.

Nesses estudos, pode-se observar que os atributos físicos do solo têm se relacionado, com maior frequiência, à capacidade produtiva dos sítios florestais e, conseqüentemente, com a qualidade da madeira produzida (Gonçalves, Demattê \& Couto, 1990).

R. Árvore, Viçosa-MG, v.28, n.2, p.267-273, 2004
De modo geral, quanto menores a densidade global e a resistência à penetração de raízes e maiores a porosidade total, a macroporosidade e a disponibilidade de água, maiores são as taxas de crescimento dos sítios florestais (Brady, 1996; Theodorou et al., 1991).

Constatou-se também que, apesar de o Pinus ser considerado pouco exigente quanto à fertilidade do solo, níveis diferenciados de produção têm sido observados quando se plantam espécies em condições edáficas distintas (Balloni, 1984).

Devido a essa taxa de crescimento diferenciada, as propriedades da madeira também não são as mesmas, sendo importante a obtenção de informações detalhadas sobre as propriedades deste material.

O objetivo deste trabalho foi mostrar a influência dos atributos do solo (físicos e químicos) sobre a qualidade da madeira de Pinus taeda L., de forma a permitir a seleção de sítios apropriados para produção desta madeira, visando a produção de celulose.

\section{MATERIAL EMÉTODOS}

\subsection{Localização e Descrição da Área Experimental}

As amostras de madeira estudadas neste trabalho foram obtidas de árvores de Pinus taeda, provenientes de plantios com 12 anos de idade, localizados na Cia. Klabin do Paraná de Celulose S.A., em Telêmaco BorbaPR, a 240ㅇ' latitude sul e 50³0' longitude oeste, com altitude de 750 a $868 \mathrm{~m}$.

O clima da região, segundo a classificação de Köppen, é $\mathrm{Cfa} / \mathrm{Cfb}$, subtropical úmido transicional para temperado propriamente dito, em que a temperatura média do mês mais frio é inferior a $16^{\circ} \mathrm{C}$, com ocorrência de geada, e a temperatura média do mês mais quente é superior a $22^{\circ} \mathrm{C}$. A precipitação média anual é $1.490 \mathrm{~mm}$, com excedente hídrico de $557 \mathrm{~mm} / \mathrm{ano}$, distribuído em todos os meses do ano.

O material de origem da região é predominantemente relacionado ao intemperismo e retrabalhamento de litologias referentes às formações Rio Bonito, Itararé e Diques de Diabásio.

A cobertura vegetal primitiva era representada pelo contato estepe/floresta, sendo $51 \%$ formação florestal e $49 \%$ formação campo. 


\subsection{Amostragem}

Foram analisados oito sítios, selecionados pelo tipo de solo (Latossolo e Cambissolo), pela textura do solo (argilosa e média) e pela vegetação primária (campo e floresta), com base no levantamento e no mapeamento de solos existentes nas áreas de plantio da Cia. Klabin (Quadro 1).

Quadro 1 - Sítios selecionados para este estudo Table 1 -Description of selected sites

\begin{tabular}{|l|l|l|l|}
\hline \multicolumn{1}{|c|}{ Sítio } & Tipo de Solo & Textura & $\begin{array}{c}\text { Vegetação } \\
\text { Primária }\end{array}$ \\
\hline Cerradinho & Latossolo & Média & Campo \\
\hline Faisqueira & Latossolo & Argilosa & Campo \\
\hline Mandaçaia I & Latossolo & Média & Floresta \\
\hline Sete Rincões & Latossolo & Argilosa & Floresta \\
\hline Mandaçaia II & Cambissolo & Média & Campo \\
\hline Restingão & Cambissolo & Argilosa & Campo \\
\hline Campina Alta & Cambissolo & Média & Floresta \\
\hline Cirol & Cambissolo & Argilosa & Floresta \\
\hline
\end{tabular}

Devido à dificuldade de obter medidas fidedignas em relação aos atributos do solo, todos fatores do sítio que influenciam o crescimento foram cuidadosamente controlados, para se manterem constantes ou aproximadamente constantes.

\subsubsection{Amostragem do Solo e Análise de seus Atributos}

Foram coletadas amostras de três horizontes: 0-10, $10-20$ e 20-30 cm de profundidade, com três repetições, tendo sido coletadas amostras indeformadas (através de anéis volumétricos) e amostras compostas.

As amostras indeformadas foram utilizadas para determinar os atributos físicos do solo: densidade global, porosidade total, macroporosidade e disponibilidade de água, seguindo metodologia preconizada pela EMBRAPA (1997).

As amostras compostas foram utilizadas para determinar os atributos químicos do solo: macroelementos, capacidade de troca de cátions e matéria-orgânica; e as análises granulométricas, foram realizadas com base na metodologia preconizada pela EMBRAPA (1997).

\subsubsection{Amostragem das Árvores e Análise da Qualidade da Madeira}

Para seleção das árvores que seriam analisadas em cada sítio, foram realizadas avaliações dendrométricas (altura e DAP) de 50 árvores por sítio e, a partir das médias obtidas nestes sítios, selecionaram-se cinco árvores medianas por sítio.

De cada árvore selecionada foram anotados a altura total, a altura comercial e o DAP, e, em seguida, foram retirados seis discos com $5 \mathrm{~cm}$ de espessura nas seguintes posições: base (altura do corte), DAP (1,30 m do solo) e a 25, 50, 75 e $100 \%$ da altura comercial (diâmetro de $8 \mathrm{~cm}$ ).

A partir desse material foram analisadas as seguintes variáveis, quanto à qualidade da madeira: densidade básica, através do método da balança hidrostática (ABTCP, 1968), características morfológicas dos traqueídeos (Barrichello \& Foelkel, 1983; Schaitza et al., 1998) e composição química (ABTCP, 1968), e, quanto à produção de celulose: o rendimento bruto, o rendimento depurado, o número Kappa e a porcentagem de rejeitos da celulose Kraft produzida (ABNT, 1998).

\subsection{Análise Estatística}

Os valores observados foram submetidos à análise de variância (ANOVA), após realizado o teste de Bartlett de homogeneidade das variâncias. Aplicou-se o teste de Tukey, a 95\% de probabilidade, para comparação de médias entre as variáveis estudadas. Além dos testes mencionados, foi analisado o modelo de regressão linear simples entre as variáveis estudadas e as possíveis relações existentes entre essas variáveis: modelo linear ( $y=a+b x)$, determinando-se os coeficientes de correlação. Para os cálculos, utilizou-se o programa "Statistica", versão 5.0

\section{RESULTADOS E DISCUSSÃO}

\subsection{Propriedades da Madeira}

\subsubsection{Densidade Básica}

Os valores médios da densidade básica da madeira não apresentaram diferenças estatísticas significativas entre os sítios estudados (Quadro 2). Os valores médios determinados neste estudo situam-se dentro da faixa de variação dos resultados encontrados por pesquisadores

R. Árvore, Viçosa-MG, v.28, n.2, p.267-273, 2004 
que trabalharam com árvores de idades próximas, como Moreschi (1976) e Tomaselli (1980).

De acordo com o Quadro 2, apesar de não haver diferenças estatísticas significativas entre os sítios estudados, de modo geral, a madeira proveniente de sítios com ritmo de crescimento elevado, sítios com textura mais argilosa, mostrou tendência a ter menores valores de densidade básica.

Este fato foi comprovado por Tsoumis \& Panagiotidis (1980), estudando o efeito das condições de crescimento nas características da madeira de Pinus nigra, quando observaram que as madeiras provenientes de sítios com ritmo de crescimento elevado, sítios com textura mais argilosa, apresentavam menores valores de densidade básica.

Quadro 2 - Valores médios da densidade básica para madeira de Pinus taeda para cada sítio

Table 2 - Basic density average values for Pinus taeda wood for each site

\begin{tabular}{|c|c|}
\hline Sítio & $\begin{array}{c}\text { Densidade Básica } \\
\left(\mathrm{g} / \mathrm{cm}^{3}\right)\end{array}$ \\
\hline Mandaçaia I & $0,394 \mathrm{a}$ \\
\hline Campina Alta & $0,391 \mathrm{a}$ \\
\hline Cerradinho & $0,386 \mathrm{a}$ \\
\hline Mandaçaia II & $0,384 \mathrm{a}$ \\
\hline Restingão & $0,381 \mathrm{a}$ \\
\hline Cirol & $0,379 \mathrm{a}$ \\
\hline Faisqueira & $0,376 \mathrm{a}$ \\
\hline Sete Rincões & $0,373 \mathrm{a}$ \\
\hline CV $(\%)$ & 4,02 \\
\hline
\end{tabular}

As médias da densidade básica, acompanhadas da mesma letra são estatisticamente iguais entre si a $95 \%$ de probabilidade, pelo Teste de Tukey.

\subsubsection{Composição Química}

Os valores médios da composição química da madeira apresentaram diferenças estatísticas significativas entre os sítios estudados (Quadro 3), tendo que, os valores médios obtidos neste estudo situado na faixa de variação dos resultados apresentados pelo IPT (1988), para as espécies do gênero Pinus plantadas no Brasil, e também aos apresentados por Rydholm (1965), para Pinus elliottii, do sul dos Estados Unidos.

R. Árvore, Viçosa-MG, v.28, n.2, p.267-273, 2004
Quadro 3 - Valores médios da composição química da madeira de Pinus taeda para cada sítio

Table 3 - Chemical composition average values of Pinus taeda wood for each site

\begin{tabular}{|l|c|c|c|c|}
\hline \multirow{2}{*}{ Sítio } & Extrativos & Lignina & Holocelulose & Celulose \\
\cline { 2 - 5 } & \multicolumn{4}{|c|}{$(\%)$} \\
\hline Mandaçaia I & $2,93 \mathrm{a}$ & $27,22 \mathrm{ab}$ & $69,85 \mathrm{a}$ & $55,80 \mathrm{a}$ \\
\hline Campina Alta & $2,95 \mathrm{a}$ & $28,16 \mathrm{bc}$ & $68,89 \mathrm{~b}$ & $55,23 \mathrm{a}$ \\
\hline Mandaçaia II & $2,97 \mathrm{a}$ & $26,29 \mathrm{a}$ & $70,34 \mathrm{a}$ & $55,82 \mathrm{a}$ \\
\hline Cerradinho & $3,00 \mathrm{a}$ & $27,71 \mathrm{abc}$ & $69,29 \mathrm{a}$ & $55,28 \mathrm{a}$ \\
\hline Cirol & $3,01 \mathrm{a}$ & $28,91 \mathrm{c}$ & $68,08 \mathrm{~b}$ & $53,81 \mathrm{c}$ \\
\hline Sete Rincões & $3,03 \mathrm{ab}$ & $28,31 \mathrm{c}$ & $68,66 \mathrm{~b}$ & $54,81 \mathrm{~b}$ \\
\hline Faisqueira & $3,03 \mathrm{ab}$ & $28,81 \mathrm{c}$ & $68,16 \mathrm{~b}$ & $53,92 \mathrm{c}$ \\
\hline Restingão & $3,06 \mathrm{~b}$ & $29,73 \mathrm{c}$ & $67,21 \mathrm{c}$ & $53,69 \mathrm{c}$ \\
\hline CV (\%) & 4,76 & 2,03 & 0,75 & 0,80 \\
\hline
\end{tabular}

As médias da composição química, acompanhadas da mesma letra, são estatisticamente iguais entre si a $95 \%$ de probabilidade, pelo teste de Tukey.

De modo geral, a madeira proveniente de sítios cujo ritmo de crescimento foi elevado, sítios com textura mais argilosa, apresentou menores teores de holocelulose (Quadro 3), resultando em menores rendimentos durante o processo de deslignificação (Rydholm, 1965; Wakeley, 1969; Moore \& Effland, 1974).

Tsoumis \& Panagiotidis (1980) constataram que mesmo não havendo diferenças estatísticas significativas os teores de extrativos e lignina foram maiores em sítios com maior ritmo de crescimento, sítios com textura mais argilosa, e, conseqüentemente, o rendimento do processo de obtenção de celulose foi menor.

\section{1,3. Características Morfológicas dos Traqueídeos}

Os valores médios das características morfológicas dos traqueídeos apresentaram diferenças estatísticas significativas entre os sítios estudados (Quadro 4). Os valores médios determinados situam-se dentro da faixa de variação dos resultados encontrados por pesquisadores que trabalharam com árvores de idades próximas, como Moreschi (1976) e Tomaselli (1980).

As madeiras provenientes de sítios com ritmo de crescimento elevado, sítios com textura mais argilosa, mostraram tendência a ter menores valores de densidade básica (Quadro 2), traqueídeos mais largos, mais curtos, com paredes mais finas e com maiores valores para diâmetro do lúmen (Quadro 4). Os dados possibilitam afirmar que essas madeiras tendem a ser mais permeáveis 
ao licor de cozimento. Os reagentes químicos penetram mais facilmente nos cavacos menos densos e com traqueídeos de lúmen maior (Foelkel, 1976). Em se tratando de condições de cozimento semelhantes para todos os tratamentos, esses cavacos sofrem maior ataque do licor de cozimento, simplesmente em função de sua maior área de exposição.

Em síntese, o que se pode constatar é que o ritmo de crescimento mais elevado, imposto pelos "sítios mais férteis", implicou a produção de traqueídeos mais largos,

Quadro 4 - Valores médios das características morfológicas dos traqueídeos da madeira de Pinus taeda para cada sítio

Table 4 - Morphological characteristic average values of Pinus taeda wood tracheids for each site

\begin{tabular}{|l|c|c|c|c|}
\hline \multirow{2}{*}{ Sítio } & $\begin{array}{c}\text { Compri- } \\
\text { mento }\end{array}$ & Largura & $\begin{array}{c}\text { Diâmetro } \\
\text { do Lúmen }\end{array}$ & $\begin{array}{c}\text { Espessura } \\
\text { da Parede }\end{array}$ \\
\cline { 2 - 5 } & $(\mathrm{mm})$ & \multicolumn{3}{|c|}{$(\mu \mathrm{m})$} \\
\hline Cerradinho & $3,47 \mathrm{a}$ & $42,61 \mathrm{ab}$ & $32,08 \mathrm{~b}$ & $4,60 \mathrm{a}$ \\
\hline Mandaçaia I & $3,45 \mathrm{ab}$ & $41,99 \mathrm{c}$ & $31,00 \mathrm{c}$ & $4,80 \mathrm{a}$ \\
\hline Mandaçaia II & $3,45 \mathrm{ab}$ & $42,24 \mathrm{~b}$ & $31,90 \mathrm{bc}$ & $4,70 \mathrm{a}$ \\
\hline Campina Alta & $3,43 \mathrm{ab}$ & $41,94 \mathrm{c}$ & $31,08 \mathrm{c}$ & $4,80 \mathrm{a}$ \\
\hline Sete Rincões & $3,43 \mathrm{ab}$ & $42,84 \mathrm{ab}$ & $31,95 \mathrm{bc}$ & $4,20 \mathrm{~b}$ \\
\hline Faisqueira & $3,43 \mathrm{ab}$ & $43,02 \mathrm{ab}$ & $33,09 \mathrm{ab}$ & $4,20 \mathrm{~b}$ \\
\hline Cirol & $3,38 \mathrm{~b}$ & $43,08 \mathrm{a}$ & $34,16 \mathrm{a}$ & $4,30 \mathrm{~b}$ \\
\hline Restingão & $3,38 \mathrm{~b}$ & $42,99 \mathrm{ab}$ & $34,11 \mathrm{ab}$ & $4,50 \mathrm{a}$ \\
\hline CV $(\%)$ & 1,23 & 0,65 & 0,47 & 2,02 \\
\hline
\end{tabular}

As médias das características morfológicas dos traqueídeos, acompanhadas da mesma letra, são estatisticamente iguais entre si a $95 \%$ de probabilidade, pelo teste de Tukey. mais curtos, com paredes mais finas e com maiores diâmetros do lúmen. Tais características também foram observadas por Tsoumis \& Panagiotidis (1980).

\subsubsection{Produção de Celulose Kraft}

Os valores médios das características da celulose produzida em laboratório pelo processo Kraft apresentaram diferenças estatísticas significativas entre os sítios estudados (Quadro 5).

Quadro 5 - Valores médios das características da celulose Kraft produzida com a madeira de Pinus taeda para cada sítio

Table 5 - Average values of the characteristics of Pinus taeda - produced Kraft cellulose for each site

\begin{tabular}{|l|c|c|c|c|}
\hline \multirow{2}{*}{ Sítio } & $\begin{array}{c}\text { Rendimento } \\
\text { Bruto }\end{array}$ & $\begin{array}{c}\text { Rendimento } \\
\text { Depurado }\end{array}$ & $\begin{array}{c}\text { Porcentagem } \\
\text { de Rejeitos }\end{array}$ & $\begin{array}{c}\text { Numero } \\
\text { Kappa }^{1}\end{array}$ \\
\cline { 2 - 4 } & \multicolumn{4}{|c|}{$(\%)$} \\
\hline Campina Alta & $50,97 \mathrm{a}$ & $50,69 \mathrm{a}$ & $0,284 \mathrm{a}$ & $31,47 \mathrm{c}$ \\
\hline Mandaçaia II & $50,93 \mathrm{a}$ & $50,65 \mathrm{a}$ & $0,280 \mathrm{a}$ & $31,13 \mathrm{~b}$ \\
\hline Cerradinho & $50,88 \mathrm{a}$ & $50,61 \mathrm{a}$ & $0,280 \mathrm{a}$ & $31,06 \mathrm{ab}$ \\
\hline Mandaçaia I & $49,11 \mathrm{~b}$ & $48,83 \mathrm{~b}$ & $0,280 \mathrm{a}$ & $31,05 \mathrm{ab}$ \\
\hline Cirol & $49,07 \mathrm{~b}$ & $48,79 \mathrm{~b}$ & $0,280 \mathrm{a}$ & $31,41 \mathrm{c}$ \\
\hline Restingão & $49,04 \mathrm{~b}$ & $48,76 \mathrm{~b}$ & $0,280 \mathrm{a}$ & $31,05 \mathrm{ab}$ \\
\hline Faisqueira & $48,97 \mathrm{~b}$ & $48,69 \mathrm{~b}$ & $0,268 \mathrm{a}$ & $30,85 \mathrm{a}$ \\
\hline Sete Rincões & $48,83 \mathrm{~b}$ & $48,56 \mathrm{~b}$ & $0,264 \mathrm{a}$ & $31,04 \mathrm{ab}$ \\
\hline CV (\%) & 0,35 & 0,36 & 11,47 & 20,91 \\
\hline
\end{tabular}

As médias das características da celulose kraft produzida, acompanhadas da mesma letra, são estatisticamente iguais entre si a $95 \%$ de probabilidade, pelo teste de Tukey.

1/ Admensional.

Quadro 6 - Coeficiente de correlação (r) entre os atributos físicos e químicos do solo e a qualidade da madeira de Pinus taeda para produção de celulose Kraft

Table 6 -Correlation coefficient $(r)$ among the physical and chemical attributes of the soil and Pinus taeda wood quality for Kraft cellulose production

\begin{tabular}{|c|c|c|c|c|c|}
\hline \multirow[t]{2}{*}{ Atributo do Solo } & Extrativo & Celulose & Lignina & $\begin{array}{c}\text { Rendimento } \\
\text { Bruto }\end{array}$ & $\begin{array}{l}\text { Largura do } \\
\text { Traqueídeo }\end{array}$ \\
\hline & \multicolumn{5}{|c|}{ (r) } \\
\hline $\mathrm{pH}$ & & & & $0,530 * *$ & $0,501^{*}$ \\
\hline $\mathrm{K}$ & & $-0,551 * *$ & & & \\
\hline CTC & $-0,610 * *$ & $-0,535 * *$ & & & \\
\hline Densidade global & $-0,500 *$ & & $-0,591 * *$ & & $-0,513 *$ \\
\hline Porosidade total & $0,540 * *$ & & $0,548 * *$ & $-0,613 * *$ & $0,557 * *$ \\
\hline Macroporosidade & $0,742 * *$ & & & & \\
\hline
\end{tabular}

$*$ = significativo a $95 \%$ de probabilidade e $* *=$ significativo a $99 \%$ de probabilidade. 
Observa-se que os rendimentos médios obtidos nos cozimentos experimentais situam-se dentro dos valores normais para coníferas, cuja faixa de variação em rendimento pelo processo Kraft, dependendo das condições, varia entre 40 e $50 \%$ (Smook, 1989).

Os números Kappa médios variaram dentro dos limites aceitáveis na experimentação e os teores de rejeitos foram bastante baixos, demonstrando que a espécie respondeu bem às condições de cozimento.

De modo geral, pode-se observar que as madeiras provenientes de sítios com ritmo de crescimento elevado, geralmente sítios mais argilosos, propiciaram menores rendimentos em celulose (Quadro 5), o que ocorre pelo fato de elas apresentarem menores valores de densidade básica (Quadro 2), maiores teores de extrativos e lignina e menores de holocelulose (Quadro 3), traqueídeos mais curtos, mais largos, com paredes mais finas e com diâmetros do lúmen maiores (Quadro 4).

\subsubsection{Correlações Obtidas nos Sítios Estudados}

As melhores correlações encontradas entre a qualidade da madeira e os atributos físicos e químicos do solo estão apresentadas no Quadro 6.

Porém, como não existem na literatura disponível trabalhos que expliquem tais correlações, fica nessa questão um ponto a ser explorado em pesquisas futuras. Mesmo assim pode-se constatar que a madeira produzida em sítios com ritmo de crescimento elevado, geralmente sítios mais argilosos, é de menor qualidade para produção de celulose Kraft.

\section{CONCLUSÃO}

Os atributos físicos do solo demonstraram ter grande influência nas propriedades da madeira e, conseqüentemente, no rendimento em celulose.

De modo geral, as madeiras provenientes de sítios com texturas mais argilosas apresentaram menores valores de densidade básica, maiores teores de extrativos e lignina e, conseqüentemente, menores teores de holocelulose e celulose, traqueídeos mais curtos, mais largos, com paredes mais finas e com diâmetros do lúmen maiores e, conseqüentemente, menores rendimentos em celulose.

A partir dos resultados obtidos neste trabalho, concluiu-se que é possível a previsão de determinadas propriedades da polpa celulósica através da análise das características da madeira, que são resultantes das condições edáficas reinantes.

\section{REFERÊNCIAS BIBLIOGRÁFICAS}

ASSOCIAÇÃO BRASILEIRA DE NORMAS TÉCNICAS ABNT: Normas Técnicas. Rio de Janeiro: 1998. não paginado.

ASSOCIAÇÃO BRASILEIRA TÉCNICA DE CELULOSE E PAPEL - ABTCP: Normas de ensaio. São Paulo: 1968. não paginado.

BALLONI, E. A. Efeitos da fertilização mineral sobre o desenvolvimento do P. caribaea Morelet var. bahamensis (Griseb.) Barret et Golfari em solo de cerrado do estado de São Paulo. 1984. 110 f. Dissertação (Mestrado em Engenharia Florestal) - Escola Superior de Agricultura "Luiz de Queiroz", Piracicaba, 1984.

BARRICHELLO, L. E. G.; FOELKEL, C. E. B. Processo nítrico-acético para maceração de madeira. In: CONGRESSO FLORESTAL BRASILEIRO, 4., 1983, Belo Horizonte. Anais... São Paulo: Sociedade Brasileira de Silvicultura, 1983. p. 732-733.

BIRKLAND, R. Efficient process for quality products. In: IUFRO WORLD CONGRESS, 19., 1990, S.I.

Proceedings... [S.I.: s.n.], 1990. p. 139-147.

BRADY, N. C. The nature and properties of soils. 11.ed. Upper Saddle River: Prentice Hall, 1996. 639 p.

CLARKE, C. R. E.; WESSELS, A. M. Variation and measurements of pulp properties in eucalyptus. In: IUFRO WORLD CONGRESS, 1995, Hobart. Proceedings... Hobart: s.n., 1995. p. 42-44.

EMPRESA BRASILEIRA DE PESQUISA

AGROPECUÁRIA - EMBRAPA. Centro Nacional de Pesquisa de Solos. Manual de métodos de análise de solos. 2. ed. Rio de Janeiro: 1997. 212 p.

FOELKEL, C. E. B. Celulose Kraft de madeira juvenil e adulta de Pinus elliottii. Boletim Técnico IPEF, v. 12, p. 127-142, 1976.

GONÇALVES, J. L. M.; DEMATTÊ, J. L. I.; COUTO, H. T.Z. Relações entre a produtividade de sítios florestais de Pinus taeda e Pinus elliottii com as propriedades de alguns solos de textura arenosa e média no Estado de São Paulo. Boletim Técnico IPEF, n. 43/44, p. 24-39, 1990.

INSTITUTO DE PESQUISAS TECNOLÓGICAS - IPT. Celulose e papel. 2. ed. São Paulo: 1988. v. 1. 113 p. 
MOORE, W. E.; EFFLAND, M. Chemical composition of fast growth juvenil wood and slow-growth mature sycamore and cotton-wood. Tappi Journal, v. 57, n. 8, p. 96-98, 1974.

MORESCHI, J. C. Levantamento da qualidade da madeira com plantações artificiais de Pinus elliottii nos estados do sul do Brasil. 1976. 162 f. Dissertação (Mestrado em Ciências Florestais) - Universidade Federal do Paraná, Curitiba, 1976.

RYDHOLM, S. A. Pulping processes. London: Interscience, 1965. $1269 \mathrm{p}$.

SANSÍGOLO, C. A. Seleção de árvores matrizes para a produção de polpa Kraft-AQ. 2000. 157 f. Tese (Livre Docência) - Universidade Estadual Paulista, Botucatu, 2000.

SCHAITZA, E. G.; MATTOS, P. P.; PEREIRA, J. C. D. Metodologías sencillas y baratas para análisis de imagem en laboratorios de calidad de madera. In: CONGRESSO LATINOAMERICANO IUFRO, 1., 1998, Valdivia. El manejo sustentable de los recursos forestales, desafio del siglo XXI: acta. Valdivia:> 1998. CD Rom.
SMOOK, G. A. Handbook for pulp and paper technologists. Joint Textbook. Committee of the Paper Industry. Tappi Journal, v. 72, n. 7, p. 235-246, 1989.

THEODOROU, C.; CAMERON, J. N.; BOWEN, G. D. Growth of roots of differents Pinus radiata genotypes at different strength and aeration. Australian Forestry Resources, v. 54, n. 12, p. 52-59, 1991.

TOMASELLI, I. Comparação da qualidade da madeira de Araucaria angustifolia e Pinus spp. produzida em reflorestamento. In: PESQUISAS em recursos florestais do Estado do Paraná. Relatório Final. Curitiba: FINEP/ UFPR, 1980.

TSOUMIS, G.; PANAGIOTIDIS, N. Effect of growth condition on quality characteristics of Black Pine (Pinus nigra Arn.) Wood Science and Technology, v. 14, p. 301310, 1980.

WAKELEY, P. C. Single commercial thinnings in variously spaced slash and loblolly pine plantations. [S.1. s.n.], 1969. 12 p. (Forestry Service Research Paper, 50) 